\title{
OPTIMISATION OF TRAFFIC LIGHT CONTROL SYSTEM USING FUZZY LOGIC AND SET THEORY
}

\author{
Stephy $\mathbf{J}^{1}$, Arabinda Roy ${ }^{2}$ \\ ${ }^{1} P G$, Department of Electronics and Telecommunication, IIEST, Shibpur, India \\ ${ }^{2}$ Visiting Prof, Department of Electronics and Telecommunication, IIEST, Shibpur, India
}

\begin{abstract}
Traffic congestion is a major problem in cities. While solving this problem, it is necessary to consider the type of the road and that of the traffic intersection. This paper considers both the lane based and non-lane based roads into account. Traffic density of each road is measured by using infrared sensors. This work introduces a simple and novel algorithm to control traffic lights. Controlling of traffic lights involves two major tasks. One is to decide the next green signal which is to be on; and the other is assigning the time duration for the selected green light. In this paper these tasks are done by using set theory and fuzzy logic.
\end{abstract}

Keywords: Traffic Density, Traffic Congestion, Intersection of Roads, Fuzzy Logic, Set Theory.

****

\section{INTRODUCTION}

Traffic congestion plays an important role in the health, environmental, economic and social issues of the cities in a country. Construction of new roads, bypass roads, flyovers and rings can partially fulfill the goal of congestion less transportation [1]. But the existing conventional traffic lights are yet to be changed, since they work under prefixed logic and they are not real time systems. The on-time duration of green lights and the sequence of selection of the next green light are fixed in the conventional system.

But the density of traffic on road depends on several parameters. The traffic density varies based on the time of the day, day of the week, time of the year and the location of the traffic junction. To control the heavy traffic congestion and to minimize the waiting time at the intersection, we need a simple, economic, real time and automatic system. Density based smart traffic light control system serves this purpose.

Design of smart traffic control system is an active research topic and it is a need of the hour. It involves several tasks such as, measurement of traffic density on each road, comparisons of the measured density, developing algorithm based on the comparison and programming a microcontroller to control the traffic light as per the algorithm. All these tasks are done by considering the type of the road, since there are mono directional non-lane based roads and multi-directional lane-based roads in the cities.

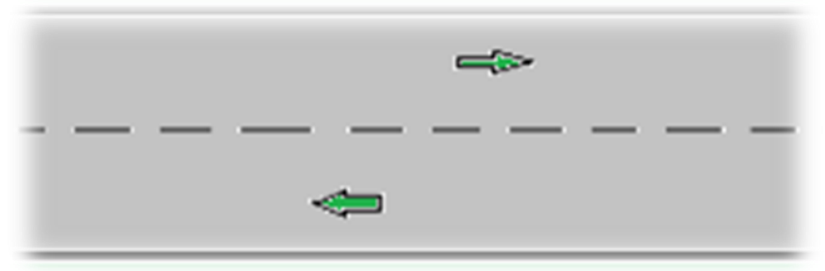

Fig-1: Non-lane based road (mono-directional)

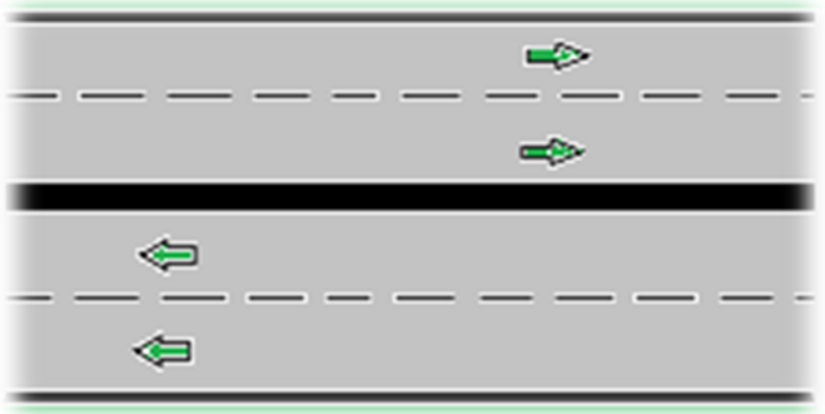

Fig-2: Lane based road (multi directional)

Infrared (IR) sensors are used to find the traffic density. The traffic congestion is classified into three types, based on this traffic density. They are: normal traffic, medium traffic and extreme traffic [2]. This density measurement is done for all the roads except the one which is currently getting the green signal. The road in which the extreme traffic is noticed will be given opportunity to get the next green signal. If no road is extremely congested, the road which has medium traffic congestion will be chosen to provide the next green signal. If more than one road has same congestion status, then preference is given to the next heavily congested road in sequential order.

Thus in the proposed algorithm, the sequence of green signal is partially random and partially sequential. The on time duration also is assigned by considering the congestion status of all the roads. This algorithm is verified using MATLAB. Microcontroller is used to control the traffic lights and Microc Pro is used to program the microcontroller. 


\section{SMART TRAFFIC LIGHT CONTROL}

\section{SYSTEM}

The concept of using sensors to control traffic light, and density based traffic control is not a new one. Many authors have written on this concept and still it is an area in which researches are going on.

Some of the authors use image processing and video processing techniques to count the number of vehicles in the queue near the intersection [3-6]. But the camera should be of good quality to get clear and noiseless image. It will increase the cost. The initial and maintenance cost is also high. Also, it is sensitive to the weather condition.

IR sensors are used to find the traffic density, in some papers [1][7-11][15]. Some of the papers use ultrasonic sensors for that purpose [12] and some other use inductive loop sensors [2]. RFID is also used to count the number of vehicles in the queue [13].

Many of the literatures consider only the mono-directional non-lane based roads in their work [1-3,5-11]. Some of the papers calculate the average waiting time, by counting the number of vehicles in the queue with the assumption that, all the vehicles are of same length and speed [14]. But they face a lot of practical difficulties.

After computing the traffic density using sensors, only the on time duration of green light is varied in some papers [1$3][13,15]$. In some other papers only the green light selection sequence is varied. But it is necessary to vary both of them, based on the traffic density.

There are some literatures that use fuzzy logic [16] and genetic algorithm. But most of them have considered only mono-directional non-lane based roads. So, we need a system which is simple, cost effective, real time based; and it should consider both the non-lane based and lane based roads.

\section{PROPOSED ALGORITHM AND DESIGN}

The block diagram of the proposed methodology is shown in figure 3 .

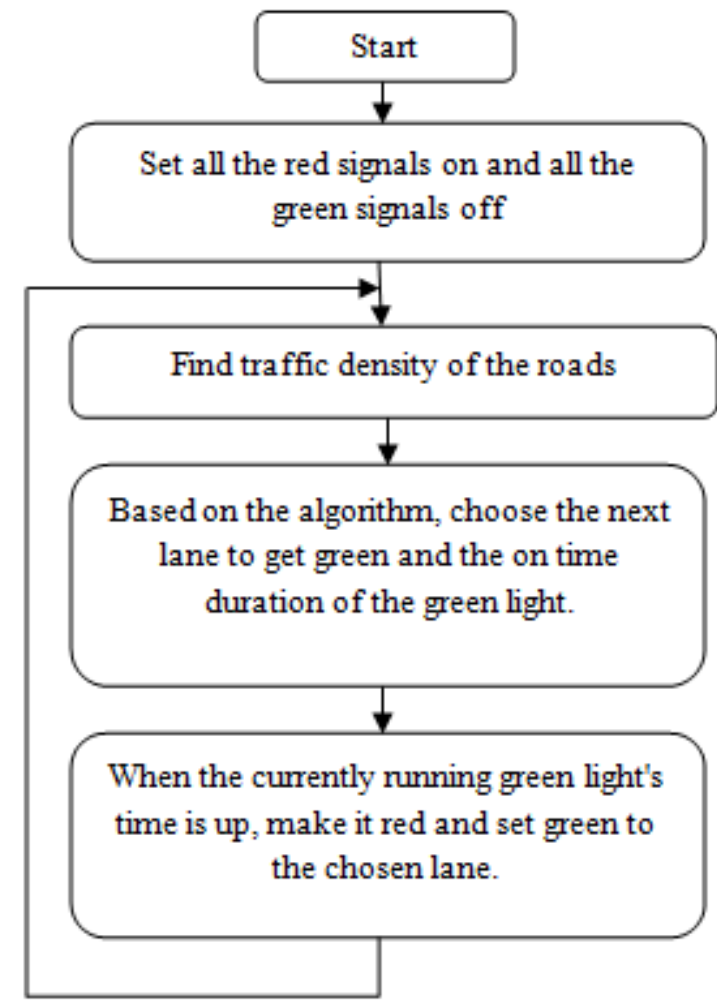

Fig-3: Block diagram of the methodology

Two IR proximity sensors are placed beside the road apart from each other by a distance. The basic idea of detecting traffic is that, when a vehicle comes near to the sensor, the IR signal gets reflected by the vehicle and gets received by the receiver. Now the proximity sensor produces high output.

The traffic density is classified into three such as normal traffic, medium traffic and extreme traffic as shown in the figure 4 .

Numbering of lanes as shown in figure 5 is necessary for convenience. The algorithm is different for lane based and non-lane based roads.

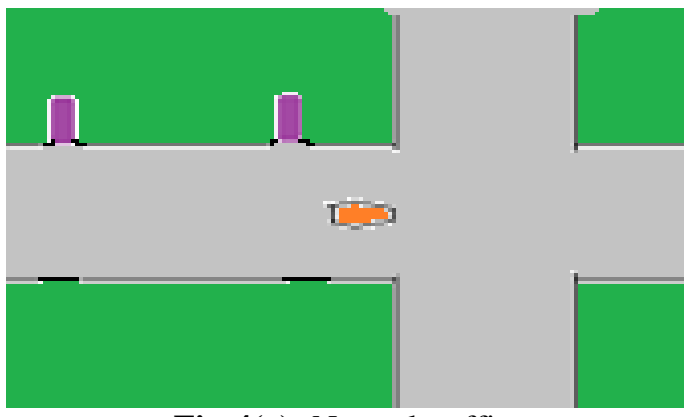

Fig-4(a): Normal traffic 


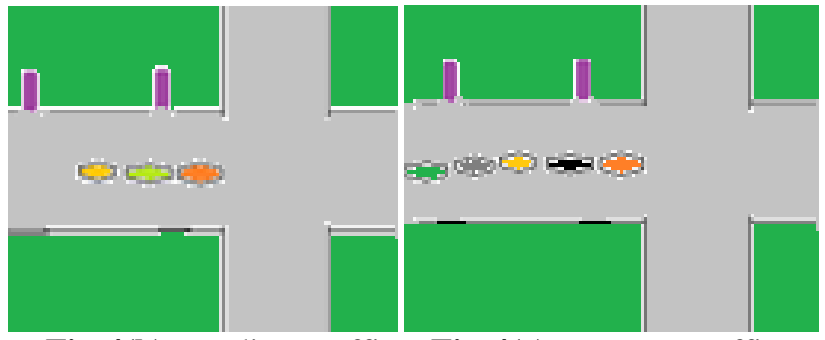

Fig-4(b): Medium traffic Fig-4(c): Extreme traffic

\subsection{Type 1: A Non Lane Based Four Road}

\section{Approaching Intersection}

Since these roads are mono-directional, the conventional three colored traffic light is sufficient. The green right turning signal is not needed.

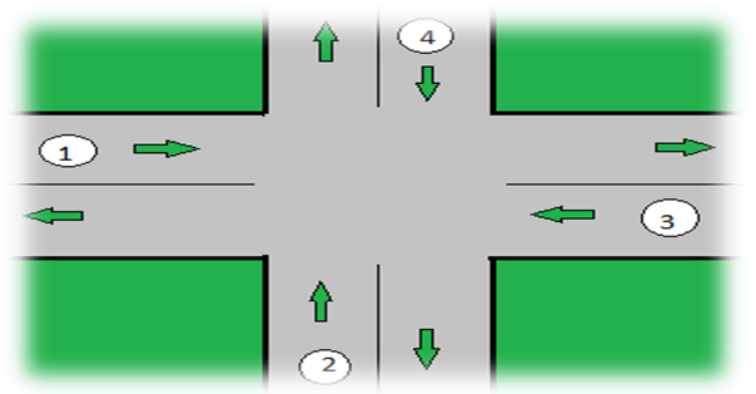

Fig-5: Numbering of roads

For the sake of explanation, let us consider that, the road-1 is currently getting green signal and it is going to become red soon. Now the traffic densities of the other roads are to be considered. The 27 possible combinations of different density status are given in table.1, in which, $\mathrm{N}$ stands for normal, $\mathrm{M}$ stands for medium and $\mathrm{E}$ for Extreme.

If the density status is such that, road-2 is medium congested and road-3 and road-4 are extremely congested, then the green light of road-3 is being activated. Now to assign the on time duration of the green light, it is necessary to consider that, there is one more road (road-4) which is extremely congested. If the maximum time is assigned to road-3, then road-4 will get congested more and more at each second. By considering all these aspects into account, this fuzzy based algorithm is developed. And the algorithm for the first type is given in table.2, in which the term 'next' indicates the normal sequence, which is the sequence of roads as numbered in figure.5.

The third column of table. 2 depicts the on time duration. In which, ' $R$ ' is a non-zero integer whose value depends on the time of the day and the location of proximity sensors. $\mathrm{R}$ is less $(\mathrm{R}=1$ or 2$)$ for non peak hours and during peak hours it is high $(\mathrm{R}>3)$. It is assigned by the code and can be adjusted by the software.

Table-1: Different combinations of traffic densities

\begin{tabular}{|l|l|l|}
\hline Road- 2 & Road- 3 & Road- 4 \\
\hline N & N & N \\
\hline
\end{tabular}

\begin{tabular}{|c|c|c|}
\hline $\mathrm{N}$ & $\mathrm{N}$ & $\mathrm{M}$ \\
\hline $\mathrm{N}$ & $\mathrm{N}$ & $\mathrm{E}$ \\
\hline $\mathrm{N}$ & $\mathrm{M}$ & $\mathrm{N}$ \\
\hline $\mathrm{N}$ & $\mathrm{M}$ & $\mathrm{M}$ \\
\hline $\mathrm{N}$ & $\mathrm{M}$ & $E$ \\
\hline $\mathrm{N}$ & $\mathrm{E}$ & $\mathrm{N}$ \\
\hline $\mathrm{N}$ & $\mathrm{E}$ & $\mathrm{M}$ \\
\hline $\mathrm{N}$ & $\mathrm{E}$ & $\mathrm{E}$ \\
\hline $\mathrm{M}$ & $\mathrm{N}$ & $\mathrm{N}$ \\
\hline $\mathrm{M}$ & $\mathrm{N}$ & $\mathrm{M}$ \\
\hline $\mathrm{M}$ & $\mathrm{N}$ & $\mathrm{E}$ \\
\hline $\mathrm{M}$ & $\mathrm{M}$ & $\mathrm{N}$ \\
\hline $\mathrm{M}$ & $\mathrm{M}$ & $\mathrm{M}$ \\
\hline $\mathrm{M}$ & $\mathrm{M}$ & $\mathrm{E}$ \\
\hline $\mathrm{M}$ & $\mathrm{E}$ & $\mathrm{N}$ \\
\hline $\mathrm{M}$ & $\mathrm{E}$ & $\mathrm{M}$ \\
\hline $\mathrm{M}$ & $\mathrm{E}$ & $E$ \\
\hline $\mathrm{E}$ & $\mathrm{N}$ & $\mathrm{N}$ \\
\hline $\mathrm{E}$ & $\mathrm{N}$ & $\bar{M}$ \\
\hline $\mathrm{E}$ & $\mathrm{N}$ & $\mathrm{E}$ \\
\hline $\mathrm{E}$ & $\mathrm{M}$ & $\mathrm{N}$ \\
\hline $\mathrm{E}$ & $\mathrm{M}$ & $\mathrm{M}$ \\
\hline $\mathrm{E}$ & $\mathrm{M}$ & $\mathrm{E}$ \\
\hline $\mathrm{E}$ & $\mathrm{E}$ & $\mathrm{N}$ \\
\hline $\mathrm{E}$ & $\mathrm{E}$ & $\bar{M}$ \\
\hline $\mathrm{E}$ & $\mathrm{E}$ & $\mathrm{E}$ \\
\hline
\end{tabular}

Table-2: Different cases and their solutions

\begin{tabular}{|c|c|c|}
\hline CASE & $\begin{array}{l}\text { SELECTION OF } \\
\text { NEXT GREEN } \\
\text { LIGHT }\end{array}$ & $\begin{array}{l}\text { ON TIME FOR } \\
\text { GREEN LIGHT } \\
\text { (seconds) }\end{array}$ \\
\hline $1 \mathrm{~N}, 1 \mathrm{M}, 1 \mathrm{E}$ & $\begin{array}{l}\text { Select the } \\
\text { Extremely } \\
\text { congested lane }\end{array}$ & $18 \times \mathrm{R}$ \\
\hline $2 \mathrm{~N}, 1 \mathrm{M}$ & $\begin{array}{l}\text { Select the } \\
\text { Medium } \\
\text { congested lane }\end{array}$ & $16 \times \mathrm{R}$ \\
\hline $2 \mathrm{~N}, 1 \mathrm{E}$ & $\begin{array}{l}\text { Select the } \\
\text { Extremely } \\
\text { congested lane }\end{array}$ & $20 \times \mathrm{R}$ \\
\hline $2 \mathrm{M}, 1 \mathrm{~N}$ & $\begin{array}{l}\text { Select the next } \\
\text { Medium } \\
\text { congested lane }\end{array}$ & $13 \times \mathrm{R}$ \\
\hline $2 \mathrm{M}, 1 \mathrm{E}$ & $\begin{array}{l}\text { Select the } \\
\text { Extremely } \\
\text { congested lane }\end{array}$ & $17 \times \mathrm{R}$ \\
\hline $2 \mathrm{E}, 1 \mathrm{~N}$ & $\begin{array}{l}\text { Select the next } \\
\text { Extremely } \\
\text { congested lane }\end{array}$ & $15 \times \mathrm{R}$ \\
\hline $2 \mathrm{E}, 1 \mathrm{M}$ & $\begin{array}{l}\text { Select the next } \\
\text { Extremely } \\
\text { congested lane }\end{array}$ & $13 \times \mathrm{R}$ \\
\hline
\end{tabular}




\begin{tabular}{|l|l|l|}
\hline $3 \mathrm{~N}$ & $\begin{array}{l}\text { Select the next } \\
\text { lane }\end{array}$ & $5 \times \mathrm{R}$ \\
\hline $3 \mathrm{M}$ & $\begin{array}{l}\text { Select the next } \\
\text { lane }\end{array}$ & $10 \times \mathrm{R}$ \\
\hline $3 \mathrm{E}$ & $\begin{array}{l}\text { Select the next } \\
\text { lane }\end{array}$ & $12 \times \mathrm{R}$ \\
\hline
\end{tabular}

\subsection{Type 2: A Lane Based Four Road Approaching}

\section{Intersection}

In big cities most of the roads are four ways and six ways. In these types of roads, each road is divided into lanes. For example, in four way roads, two lanes approach the intersection. Among them one is used by the forward going vehicles and the other by the vehicles which are going to turn right. For these multi-directional roads, numbering is done for every lane that approaches the intersection as shown in figure 6.

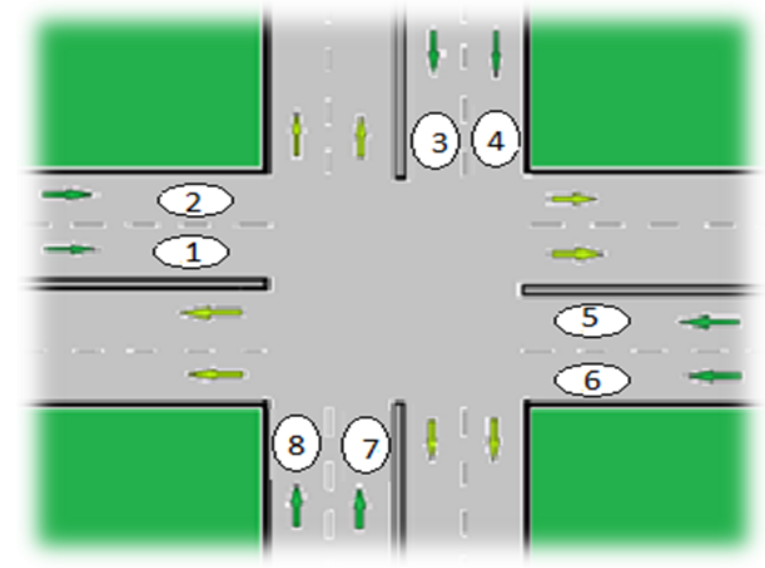

Fig-6: Numbering of lanes of multi directional roads

Along with the three colored conventional traffic signal, the right turning green signal is also used for this type. The possible traffic flows are shown in Fig.7, in which the numbers inside the braces indicates the lanes which get green signal simultaneously.

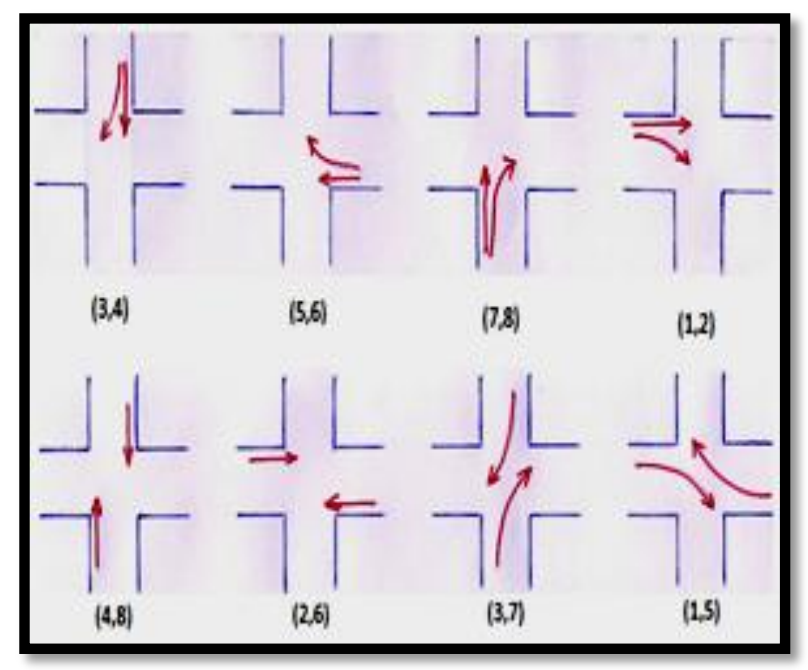

Fig-7: Possible traffic flows
All the considered traffic flows are a combination of two flows. If the combination $(1,2)$ is currently getting green signal and going to become red, the traffic density of the lanes $3,4,5,6,7$, and 8 are checked. By considering normal, medium and extreme traffic densities for each lane we will get 729 combinations.

This problem is solved by using set theory and fuzzy logic. It can be explained as follows:

Consider

a set $\mathrm{A}=\{(2,6),(4,8),(1,2),(3,4),(5,6),(7,8),(1,5),(3,7)\}$, which is the set of all possible combinations of traffic flow. Let $(a, b)$ $=(b, a)$ in the set.

If $(a, b)$ is currently green and it is going to become red, we need to choose the next combination. While choosing the next combination, the elements of set $\mathrm{A}$ which contain (a and b) and ( $\mathrm{a}$ or b) are excluded. Now, the new set is B.

$\mathrm{B}=\mathrm{A}-(\mathrm{elements}$ of set $\mathrm{A}$ containing $(\mathrm{a}$ and $\mathrm{b})$ and $(\mathrm{a}$ or $\mathrm{b})$ )

The solution for the second type is given in table.3, in which M-lane stands for the medium congested lane and E-lane for extremely congested lane. The first column of this table indicates the density status of six lanes. For example, 1N, $2 \mathrm{M}, 3 \mathrm{E}$ means, among the six lanes one lane has normal congestion, two lanes are medium congested and three lanes are extremely congested. Here, ' $\mathrm{R}$ ' is a non-zero integer.

Table-3: Possible cases and their solutions

\begin{tabular}{|c|c|c|c|}
\hline & $\begin{array}{l}\text { CA } \\
\text { SE }\end{array}$ & $\begin{array}{l}\text { SELECTION OF NEXT PAIR } \\
\text { OF GREEN LIGHT }\end{array}$ & $\begin{array}{l}\text { TI } \\
\text { ME } \\
\text { LI } \\
\text { MI } \\
\text { T } \\
\text { (se } \\
\text { c) }\end{array}$ \\
\hline 1 & $\begin{array}{l}\text { All } \\
\text { Nor } \\
\text { mal }\end{array}$ & Select the next element from set B & $\begin{array}{l}2 \times \\
R\end{array}$ \\
\hline 2 & $\begin{array}{l}\text { All } \\
\text { Me } \\
\text { diu } \\
\mathrm{m}\end{array}$ & Select the next element from set B & $\begin{array}{l}4 \times \\
\mathrm{R}\end{array}$ \\
\hline 3 & $\begin{array}{l}\text { All } \\
\text { Ext } \\
\text { rem } \\
\mathrm{e}\end{array}$ & Select the next element from set B & $\begin{array}{l}6 \times \\
\mathrm{R}\end{array}$ \\
\hline 4 & $\begin{array}{l}5 \mathrm{~N} \\
1 \mathrm{M}\end{array}$ & $\begin{array}{l}\text { 1. Let the variable ' } x \text { ' represent } \\
\text { the M-lane } \\
\text { 2. Select the first element of set } \\
\text { B containing ' } x \text { ' }\end{array}$ & $\begin{array}{l}6 \times \\
\mathrm{R}\end{array}$ \\
\hline 5 & $\begin{array}{l}5 \mathrm{~N}, \\
1 \mathrm{E}\end{array}$ & $\begin{array}{l}\text { 1. Let the variable ' } x \text { ' represent } \\
\text { the M-lane } \\
\text { 2. Select the first element of set } \\
B \text { containing ' } x \text { ' }\end{array}$ & $\begin{array}{l}10 \\
\times \mathrm{R}\end{array}$ \\
\hline
\end{tabular}




\begin{tabular}{|c|c|c|c|}
\hline 6 & $\begin{array}{l}4 \mathrm{~N} \\
2 \mathrm{M}\end{array}$ & $\begin{array}{l}\text { 1. Let the variables ' } x \text { ' and 'y' } \\
\text { represent the M-lanes } \\
\text { 2. If }(x, y) \text { is in set } B \text {, select it } \\
\text { 3. Else select the first element } \\
\text { with } x \text { or y from B }\end{array}$ & $\begin{array}{l}8 x \\
R \\
6 x \\
R\end{array}$ \\
\hline 7 & $\begin{array}{l}4 \mathrm{~N} \\
2 \mathrm{E}\end{array}$ & $\begin{array}{l}\text { 1. Let the variables ' } x \text { ' and ' } y \text { ' } \\
\text { represent the E-lanes } \\
\text { 2. If }(x, y) \text { is in set } B \text {, select it } \\
\text { 3. Else select the first element } \\
\text { with } x \text { or y from B }\end{array}$ & $\begin{array}{c}12 \\
\times \mathrm{R} \\
10 \\
\times \mathrm{R}\end{array}$ \\
\hline 8 & $\begin{array}{l}4 \mathrm{~N}, \\
1 \mathrm{M}, \\
1 \mathrm{E}\end{array}$ & $\begin{array}{l}\text { 1. Let ' } x \text { ' represent the E-lane } \\
\text { and ' } y \text { ' represent the M-lane } \\
\text { 2. If }(x, y) \text { is in set } B \text {, select it. } \\
\text { 3. Else select the first element } \\
\text { of B containing ' } x \text { ' }\end{array}$ & $\begin{array}{c}12 \\
\times \mathrm{R} \\
10 \\
\times \mathrm{R}\end{array}$ \\
\hline 9 & $\begin{array}{l}3 \mathrm{~N} \\
3 \mathrm{M}\end{array}$ & $\begin{array}{l}\text { Let the variables } \mathrm{x}, \mathrm{y}, \mathrm{z} \\
\text { represent the M-lanes. } \\
\text { 2. } \begin{array}{l}\text { Consider } \mathrm{a} \\
\mathrm{C}=\{(\mathrm{x}, \mathrm{y}),(\mathrm{y}, \mathrm{z}),(\mathrm{x}, \mathrm{z})\}\end{array} \\
\text { 3. Find } \mathrm{D}=\mathrm{B} \cap \mathrm{C} \\
\text { 4. If } \mathrm{D} \neq \Phi, \text { select the first } \\
\text { element in } \mathrm{D} \text {. } \\
\text { 5. Else select the first element } \\
\text { of } \mathrm{B} \text { with } \mathrm{x} \text { or } \mathrm{y} \text { or } \mathrm{z} .\end{array}$ & $\begin{array}{l}8 \times \\
R \\
6 x \\
R\end{array}$ \\
\hline 10 & $\begin{array}{l}3 \mathrm{~N} \\
3 \mathrm{E}\end{array}$ & $\begin{array}{l}\text { 1. Let the variables } \mathrm{x}, \mathrm{y}, \mathrm{z} \\
\text { represent the E-lanes. } \\
\text { Consider set } \\
\mathrm{C}=\{(\mathrm{x}, \mathrm{y}),(\mathrm{y}, \mathrm{z}),(\mathrm{x}, \mathrm{z})\} \\
\text { 3. Find } \mathrm{D}=\mathrm{B} \cap \mathrm{C} \\
\text { 4. If } \mathrm{D} \neq \Phi, \text { select the first } \\
\text { element in } \mathrm{D} \text {. } \\
\text { 5. Else select the first element } \\
\text { of } \mathrm{B} \text { with } \mathrm{x} \text { or } \mathrm{y} \text { or } \mathrm{z} .\end{array}$ & $\begin{array}{l}11 \\
\times \mathrm{R} \\
9 \times \\
\mathrm{R}\end{array}$ \\
\hline 11 & $\begin{array}{l}3 \mathrm{~N}, \\
2 \mathrm{M}, \\
1 \mathrm{E}\end{array}$ & $\begin{array}{l}\text { 1. Let ' } \mathrm{x} \text { ' represent E-lane, and } \\
\text { ' } \mathrm{y} \text { ' and ' } \mathrm{z} \text { ' represent } \mathrm{M}- \\
\text { lanes. } \\
\text { 2. } \begin{array}{l}\text { Consider } \\
\mathrm{C}=\{(\mathrm{x}, \mathrm{y}),(\mathrm{x}, \mathrm{z})\}\end{array} \\
\text { 3. Find } \mathrm{D}=\mathrm{B} \cap \mathrm{C} \\
\text { 4. If } \mathrm{D} \neq \Phi, \text { select the first } \\
\text { element in } \mathrm{D} \\
\text { 5. Else select the first element } \\
\text { of } \mathrm{B} \text { containing ' } \mathrm{x} \text { '. }\end{array}$ & $\begin{array}{c}10 \\
\times \mathrm{R} \\
11 \\
\times \mathrm{R}\end{array}$ \\
\hline 12 & $\begin{array}{l}3 \mathrm{~N} \\
1 \mathrm{M} \\
2 \mathrm{E}\end{array}$ & $\begin{array}{l}\text { 1. Let ' } \mathrm{x} \text { ' and ' } \mathrm{y} \text { ' represent } \mathrm{E}- \\
\text { lane, and ' } \mathrm{z} \text { ' represent } \mathrm{M} \text { - } \\
\text { lane } \\
\text { 20nsider } \\
\mathrm{C}=\{(\mathrm{x}, \mathrm{z}),(\mathrm{y}, \mathrm{z})\} \\
\text { 3. Find } \mathrm{D}=\mathrm{B} \cap \mathrm{C} \\
\text { 4. If }(\mathrm{x}, \mathrm{y}) \text { belongs to } \mathrm{B} \text {, then } \\
\text { select it } \\
\text { 5. Else if } \mathrm{D} \neq \Phi \text {, select the first } \\
\text { element in } \mathrm{D}\end{array}$ & $\begin{array}{l}11 \\
\times \mathrm{R} \\
10 \\
\times \mathrm{R} \\
8 \times \\
\mathrm{R}\end{array}$ \\
\hline
\end{tabular}

\begin{tabular}{|c|c|c|c|}
\hline 13 & $\begin{array}{l}2 \mathrm{~N} \\
4 \mathrm{M}\end{array}$ & $\begin{array}{ll}\text { 1. } & \text { Let 'p','q','r','s' represent } \\
& \text { M-lanes } \\
\text { 2. } & \text { Consider set } \\
& \mathrm{C}=\{(\mathrm{p}, \mathrm{q}),(\mathrm{p}, \mathrm{r}),(\mathrm{p}, \mathrm{s}),(\mathrm{q}, \mathrm{r}),(\mathrm{q}, \mathrm{s}) \\
& ,(\mathrm{r}, \mathrm{s})\} \\
\text { 3. } & \text { Find } \mathrm{D}=\mathrm{B} \cap \mathrm{C} \\
\text { 4. } & \text { Select the first element in } \mathrm{D}\end{array}$ & $\begin{array}{l}8 x \\
R\end{array}$ \\
\hline 14 & $\begin{array}{l}2 \mathrm{~N}, \\
4 \mathrm{E}\end{array}$ & $\begin{array}{ll}\text { 1. } & \text { Let 'p','q','r','s' represent } \\
& \text { E-lanes } \\
\text { 2. } & \text { Consider } \quad \text { a } \\
& \mathrm{C}=\{(\mathrm{p}, \mathrm{q}),(\mathrm{p}, \mathrm{r}),(\mathrm{p}, \mathrm{s}),(\mathrm{q}, \mathrm{r}),(\mathrm{q}, \mathrm{s}) \\
& ,(\mathrm{r}, \mathrm{s})\} \\
\text { 3. } & \text { Find } \mathrm{D}=\mathrm{B} \cap \mathrm{C} \\
\text { 4. } & \text { Select the first element in } \mathrm{D}\end{array}$ & $\begin{array}{l}9 x \\
R\end{array}$ \\
\hline 15 & $\begin{array}{l}2 \mathrm{~N}, \\
3 \mathrm{M}, \\
1 \mathrm{E}\end{array}$ & $\begin{array}{l}\text { 1. Let 'p' represents E-lane, } \\
\text { 'q','r','s' represent M-lane } \\
\text { 2. Consider a set } \\
\mathrm{C}=\{(\mathrm{p}, \mathrm{q}),(\mathrm{p}, \mathrm{r}),(\mathrm{p}, \mathrm{s})\} \\
\text { 3. Find } \mathrm{D}=\mathrm{B} \cap \mathrm{C} \\
\text { 4. If } \mathrm{D} \neq \Phi, \text { select the first } \\
\text { element in } \mathrm{D} \\
\text { 5. Else select the first element } \\
\text { of } \mathrm{B} \text { containing 'p' }\end{array}$ & $\begin{array}{l}8 \times \\
\mathrm{R} \\
7 \times \\
\mathrm{R}\end{array}$ \\
\hline 16 & $\begin{array}{l}2 \mathrm{~N}, \\
2 \mathrm{M}, \\
2 \mathrm{E}\end{array}$ & 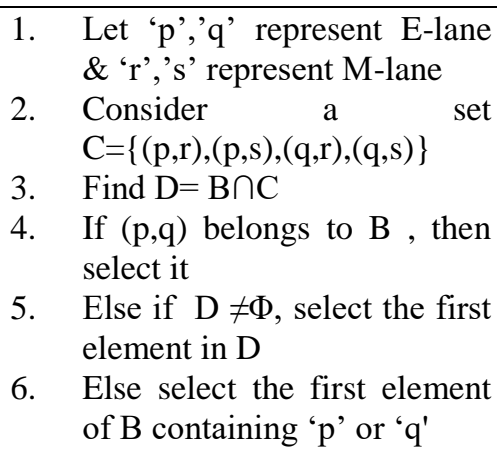 & $\begin{array}{l}10 \\
\times \mathrm{R} \\
9 \times \\
\mathrm{R} \\
7 \times \\
\mathrm{R}\end{array}$ \\
\hline 17 & $\begin{array}{l}2 \mathrm{~N}, \\
1 \mathrm{M}, \\
3 \mathrm{E}\end{array}$ & 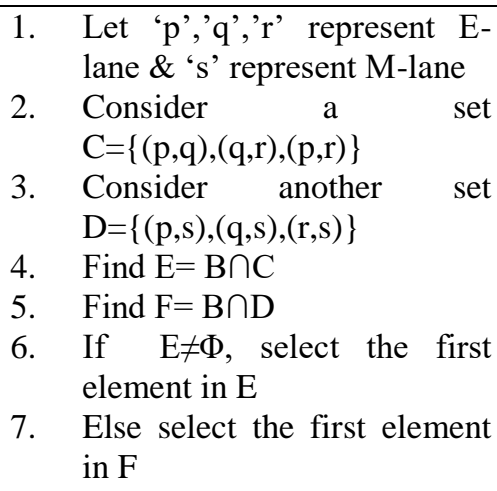 & $\begin{array}{l}10 \\
\times \mathrm{R} \\
8 \times \\
\mathrm{R}\end{array}$ \\
\hline
\end{tabular}




\begin{tabular}{|c|c|c|c|}
\hline 18 & $\begin{array}{l}1 \mathrm{~N}, \\
5 \mathrm{M}\end{array}$ & $\begin{array}{ll}\text { 1. } & \text { Let 'a','b','c','d','e' } \\
& \text { represent M-lanes } \\
\text { 2. } & \text { Consider a a } \\
& C=\{(a, b),(a, c),(a, d),(a, e),(b, c \\
& ),(b, d),(b, e),(c, d),(c, e),(d, e)\} \\
\text { 3. } & \text { Find } D=B \cap C \\
\text { 4. } & \text { Select the first element from } \\
& \text { D }\end{array}$ & $\begin{array}{l}7 \times \\
R\end{array}$ \\
\hline 19 & $\begin{array}{l}1 \mathrm{~N}, \\
5 \mathrm{E}\end{array}$ & 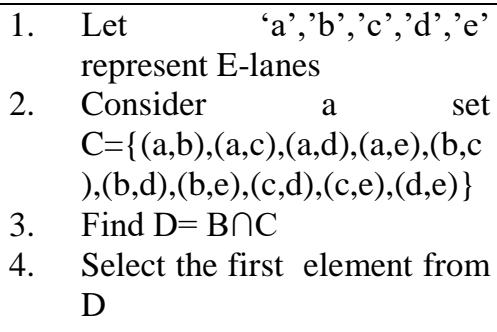 & $\begin{array}{l}8 \times \\
R\end{array}$ \\
\hline 20 & $\begin{array}{l}1 \mathrm{~N}, \\
4 \mathrm{M}, \\
1 \mathrm{E}\end{array}$ & $\begin{array}{l}\text { 1. Let 'a' represent E-lane, \& } \\
\text { 'b','c','d','e' represent M- } \\
\text { lanes } \\
\text { 2. Consider } \quad \text { a } \\
\text { C }=\{(a, b),(a, c),(a, d),(a, e)\} \\
\text { 3. Find } D=B \cap C \\
\text { 4. Select the first element from } \\
\text { D }\end{array}$ & $\begin{array}{l}10 \\
\times \mathrm{R}\end{array}$ \\
\hline 21 & $\begin{array}{l}1 \mathrm{~N}, \\
3 \mathrm{M}, \\
2 \mathrm{E}\end{array}$ & $\begin{array}{l}\text { 1. Let 'a','b' represent E-lane } \\
\& \text { 'c','d','e' represent M- } \\
\text { lane } \\
\text { Consider a a set } \\
\mathrm{C}=\{(\mathrm{a}, \mathrm{c}),(\mathrm{a}, \mathrm{d}),(\mathrm{a}, \mathrm{e})(\mathrm{b}, \mathrm{c}),(\mathrm{b}, \mathrm{d}) \\
,(\mathrm{b}, \mathrm{e})\}\end{array}$ & $\begin{array}{l}10 \\
\times \mathrm{R} \\
8 \times \\
\mathrm{R}\end{array}$ \\
\hline 22 & $\begin{array}{l}1 \mathrm{~N}, \\
2 \mathrm{M}, \\
3 \mathrm{E}\end{array}$ & 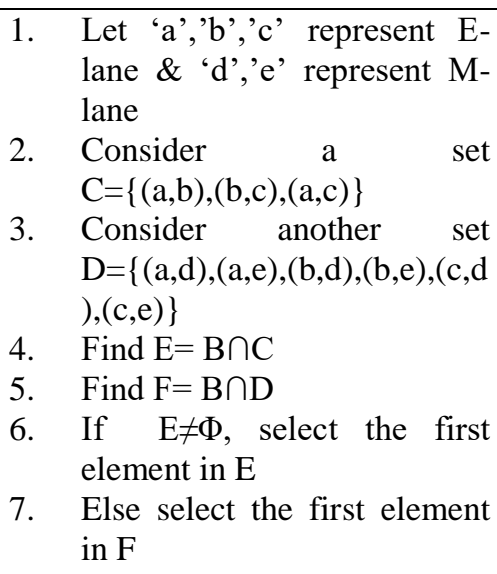 & $\begin{array}{l}9 x \\
R \\
8 x \\
R\end{array}$ \\
\hline
\end{tabular}

\begin{tabular}{|c|c|c|c|}
\hline 23 & $\begin{array}{l}1 \mathrm{~N}, \\
1 \mathrm{M}, \\
4 \mathrm{E}\end{array}$ & $\begin{array}{ll}\text { 1. Let 'a','b','c','d' represent } \\
\text { E-lanes \& 'e' represent M- } \\
\text { lane } \\
\text { 2. Consider } \quad \text { a } \\
\mathrm{C}=\{(\mathrm{a}, \mathrm{b}),(\mathrm{a}, \mathrm{c}),(\mathrm{a}, \mathrm{d}),(\mathrm{b}, \mathrm{c}),(\mathrm{b}, \mathrm{d} \\
\\
\text { 3. }(\mathrm{c}, \mathrm{d})\} \\
\text { 3. } \\
\text { Find } \mathrm{D}=\mathrm{B} \cap \mathrm{C} \\
\text { Select the first element from } \\
\mathrm{D}\end{array}$ & $\begin{array}{l}8 x \\
R\end{array}$ \\
\hline 24 & $\begin{array}{l}5 \mathrm{M}, \\
1 \mathrm{E}\end{array}$ & $\begin{array}{l}\text { 1. Let ' } x \text { ' represent E-lane } \\
\text { 2. Select the first element of } B \\
\text { containing ' } x \text { ' }\end{array}$ & $\begin{array}{r}10 \\
\times \mathrm{R}\end{array}$ \\
\hline 25 & $\begin{array}{l}4 \mathrm{M}, \\
2 \mathrm{E}\end{array}$ & $\begin{array}{l}\text { 1. Let ' } x \text { ',' } y \text { ' represent E-lanes } \\
\text { 2. If }(x, y) \text { belongs to } B \text {, select it } \\
\text { 3. Else select the first element } \\
\text { of } B \text { containing ' } x \text { ' or ' } y \text { ' }\end{array}$ & $\begin{array}{l}11 \\
\times \mathrm{R} \\
10 \\
\times \mathrm{R}\end{array}$ \\
\hline 26 & $\begin{array}{l}3 \mathrm{M}, \\
3 \mathrm{E}\end{array}$ & $\begin{array}{l}\text { 1. Let ' } x \text { ','y','z' represent E- } \\
\text { lanes } \\
\text { 2. Consider set } \\
\mathrm{C}=\{(\mathrm{x}, \mathrm{y}),(\mathrm{y}, \mathrm{z}),(\mathrm{x}, \mathrm{z})\} \\
\text { 3. Find } \mathrm{D}=\mathrm{B} \cap \mathrm{C} \\
\text { 4. If } \mathrm{D} \neq \Phi, \text { select the first } \\
\text { element in } \mathrm{D} \\
\text { 5. Else select the first element } \\
\text { in } B \text { containing ' } \mathrm{x} \text { ' or ' } \mathrm{y} \text { ' or } \\
\text { ' } \mathrm{z} \text { ' }\end{array}$ & $\begin{array}{l}10 \\
\times \mathrm{R} \\
9 \times \\
\mathrm{R}\end{array}$ \\
\hline 27 & $\begin{array}{l}2 \mathrm{M}, \\
4 \mathrm{E}\end{array}$ & $\begin{array}{ll}\text { 1. } & \text { Let 'p','q','r','s' represent } \\
& \text { E-lane } \\
\text { 2. } & \text { Consider set } \\
& \mathrm{C}=\{(\mathrm{p}, \mathrm{q}),(\mathrm{p}, \mathrm{r}),(\mathrm{p}, \mathrm{s}),(\mathrm{q}, \mathrm{r}),(\mathrm{q}, \mathrm{s}) \\
& ,(\mathrm{r}, \mathrm{s})\} \\
\text { 3. } & \text { Find } \mathrm{D}=\mathrm{B} \cap \mathrm{C} \\
\text { 4. } & \text { Select the first element in } \mathrm{D}\end{array}$ & $\begin{array}{r}10 \\
\times \mathrm{R}\end{array}$ \\
\hline 28 & $\begin{array}{l}1 \mathrm{M}, \\
5 \mathrm{E}\end{array}$ & $\begin{array}{ll}\text { 1. } & \text { Let 'a','b','c','d','e' } \\
& \text { represent E-lanes } \\
\text { 2. } & \text { Consider a a } \quad \mathrm{a} \text { set } \\
& \mathrm{C}=\{(\mathrm{a}, \mathrm{b}),(\mathrm{a}, \mathrm{c}),(\mathrm{a}, \mathrm{d}),(\mathrm{a}, \mathrm{e}),(\mathrm{b}, \mathrm{c} \\
& ),(\mathrm{b}, \mathrm{d}),(\mathrm{b}, \mathrm{e}),(\mathrm{c}, \mathrm{d}),(\mathrm{c}, \mathrm{e}),(\mathrm{d}, \mathrm{e})\} \\
\text { 3. } & \text { Find } \mathrm{D}=\mathrm{B} \cap \mathrm{C} \\
\text { 4. } & \text { Select the first element from } \\
& \mathrm{D}\end{array}$ & $\begin{array}{l}10 \\
\times \mathrm{R}\end{array}$ \\
\hline
\end{tabular}

\section{SIMULATION RESULTS}

The algorithm is verified using MATLAB. Microc Pro compiler is used to program the microcontroller and the output is simulated in Proteus simulator. 


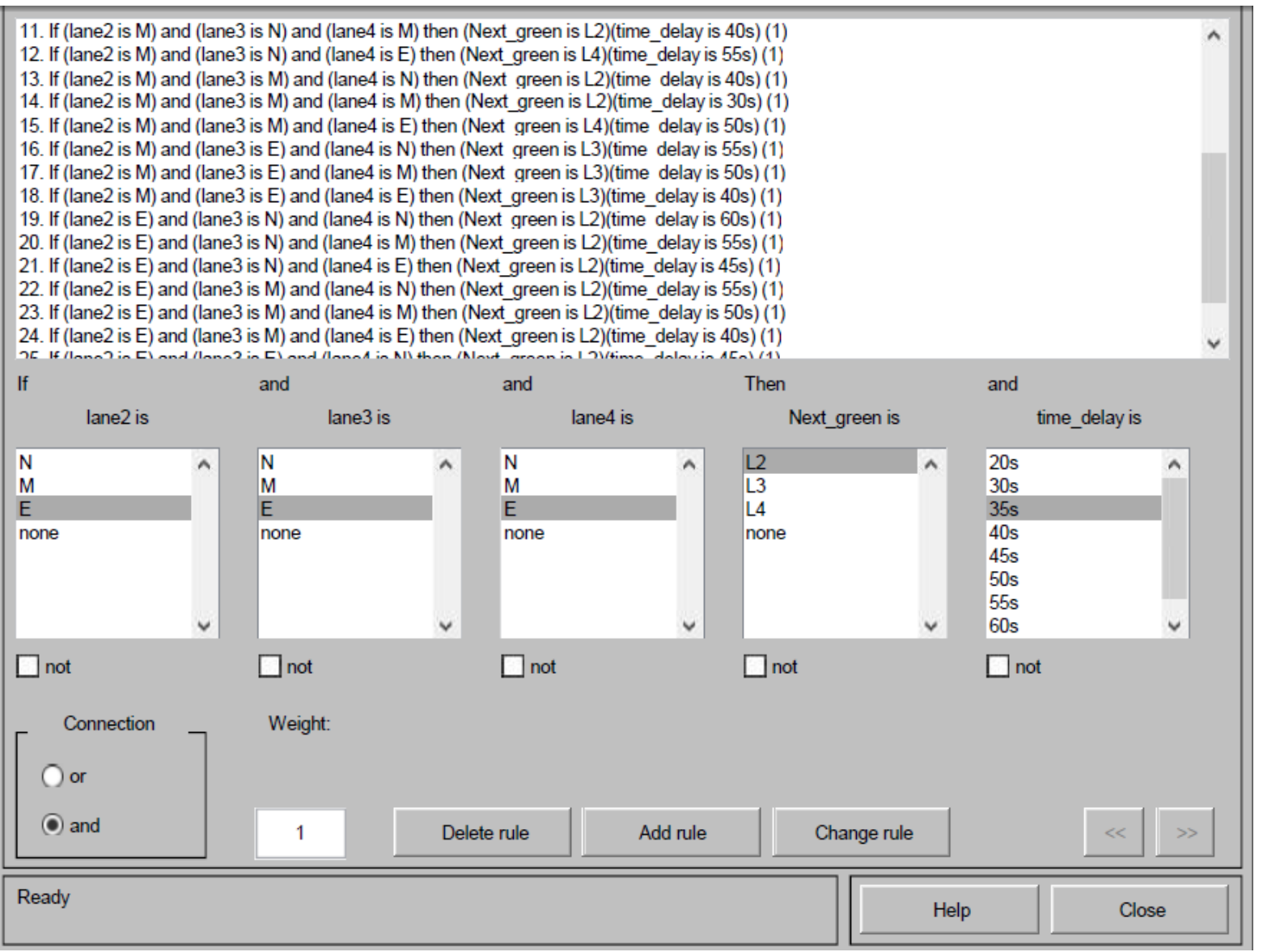

Fig-8: Verification of fuzzy logic using MATLAB

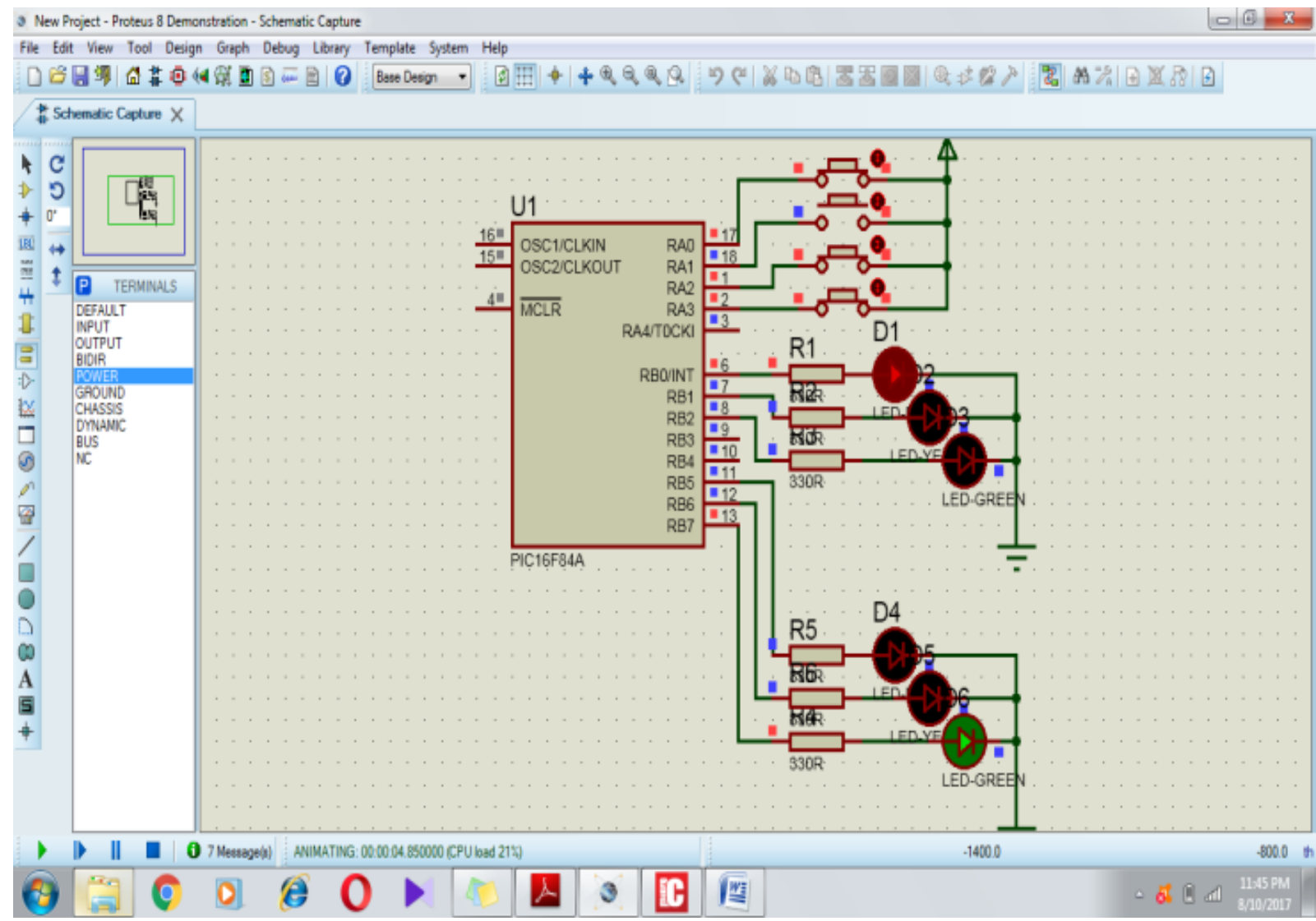

Fig-9: Simulation in Proteus software 
The major hardware used here are the microcontroller, the IR proximity sensor, the USBASP programmer and the LEDs. For experimentation purpose the microcontroller and LEDs are placed and connected on breadboard. The microcontroller is connected with the PC through USBASP programmer, and the coded program got burned into it.

The IR proximity sensor consists of both the transmitter and the receiver. A potentiometer is used to adjust the proximity length. It is shown in figure. 10.

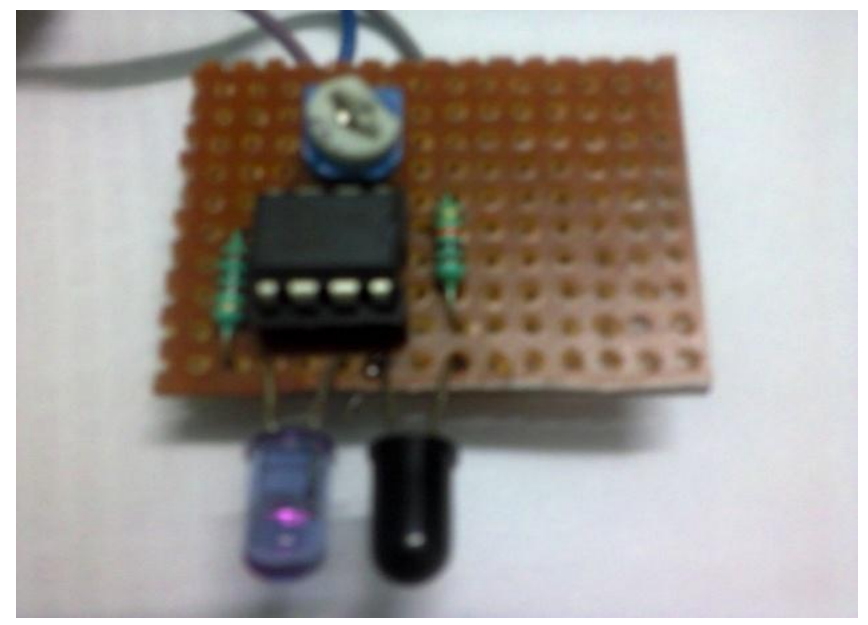

Fig-10: IR proximity sensor

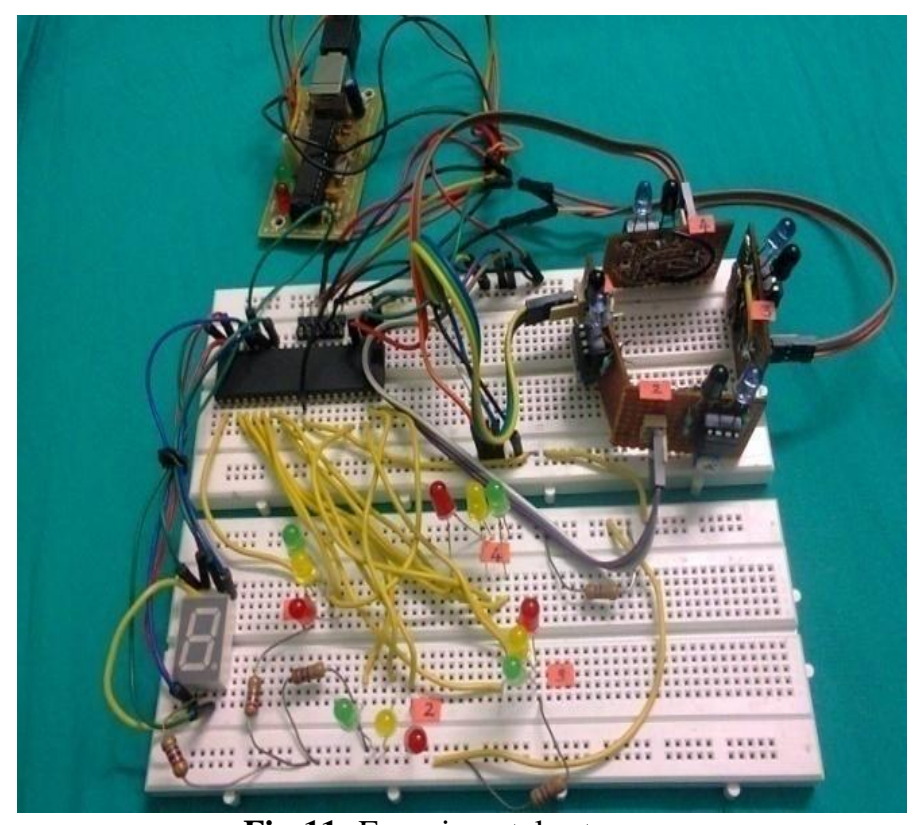

Fig-11: Experimental setup

\section{CONCLUSION}

Congestion less traffic needs updated technologies. Currently, sensors are inducted in all the fields to control the equipments. Sensors and suitable algorithms make a system smarter. The proposed fuzzy logic and set theory based algorithm works good and reduces the waiting time at the intersection. If this algorithm is used in all the traffic intersections of a city, then the total travelling time in that city will get reduced.

\section{REFERENCES}

[1] B. Ghazal, K. ElKhatib, K. Chahine and M. Kherfan, "Smart traffic light control system," 2016 Third International Conference on Electrical, Electronics, Computer Engineering and their Applications (EECEA), Beirut, 2016, pp. 140-145.

[2] L. Bhaskar, A. Sahai, D. Sinha, G. Varshney and T. Jain, "Intelligent traffic light controller using inductive loops for vehicle detection," 2015 1st International Conference on Next Generation Computing Technologies (NGCT), Dehradun, 2015, pp. 518-522.

[3] V. S. Harilakshmi and P. A. J. Rani, "Intelligent vehicle counter - a road to sustainable development and pollution prevention (P2)," 2016 International Conference on Energy Efficient Technologies for Sustainability (ICEETS), Nagercoil, 2016, pp. 877880 .

[4] D. K. Aarthy, S. Vandanaa, M. Varshini and K. Tijitha, "Automatic identification of traffic violations and theft avoidance," 2016 Second International Conference on Science Technology Engineering and Management (ICONSTEM), Chennai, 2016, pp. 7276.

[5] Nilay Mokashi, "Intelligent Traffic Signal Control Using Image Processing", International Journal Of Advance Research In Computer Science And Management Studies, Volume 3, October 2015, Issue no.10, pp. 137-143.

[6] Anurag Kanungo, Ayush Sharma "Smart Traffic Lights Switching and Traffic Density Calculation using Video Processing", RAECS UIET Panjab University Chandigarh, 06 - 08 March, 2014.

[7] G. Kavya, and B. Saranya, "Density based intelligent traffic signal system using PIC microcontroller", International journal of research in applied science \& engineering technology (IJRASET), Vol. 3, Issue I, pp. 205-209, Jan 2015.

[8] E. Geetha, V. Viswanadha, and G. Kavitha, "Design of intelligent auto traffic signal controller with emergency override", International journal of engineering science and innovative technology (IJESIT), vol. 3 , Issue 4, pp. 670-675, July 2014.

[9] A. Jadhav, B. Madhuri, and T. Ketan, "Intelligent traffic light control system (ITLCS)", Proceedings of the 4th IRF international conference, Pune, 16 March 2014.

[10] A. Dakhole, M.Moon, "Design of intelligent traffic control system based on ARM", International journal of advance research in computer science and management studies, Vol. 1, Issue 6., pp. 76-80, Nov. 2013.

[11] P.Sinhmar, "Intelligent traffic light and density control using IR sensors and microcontroller", International journal of advanced technology \& engineering research (IJATER), Vol. 2, Issue 2, pp. 30-35, March 2012.

[12] Z. Li, C. Li, Y. Zhang and X. Hu, "Intelligent traffic light control system based on real time traffic flows," 2017 14th IEEE Annual Consumer 
Communications \& Networking Conference (CCNC), Las Vegas, NV, 2017, pp. 624-625.

[13] R. Sivakumar, G. Vignesh, V. Narayanan, S. Prakash and V. Sivakumar, "Automated traffic light control system and stolen vehicle detection," 2016 IEEE International Conference on Recent Trends in Electronics, Information \& Communication Technology (RTEICT), Bangalore, 2016, pp. 15941597.

[14] B. Zhou, J. Cao, X. Zeng and H. Wu, "Adaptive Traffic Light Control in Wireless Sensor NetworkBased Intelligent Transportation System," 2010 IEEE 72nd Vehicular Technology Conference - Fall, Ottawa, ON, 2010, pp. 1-5.

[15] M. A. Kumaar, G. A. Kumar and S. M. Shyni, "Advanced traffic light control system using barrier gate and GSM," 2016 International Conference on Computation of Power, Energy Information and Commuincation (ICCPEIC), Chennai, 2016,

[16] Hamed, S.R.Hajazi,"A New Traffic Light Controller Using Fuzzy Logic for a Full Single Junction Involving Emergency Vehicle Preemption", Journal of Uncertain Systems Vol.9, No.1, pp.49-61, 2015. 